Current Opinion in Chemical Biology

\title{
High-speed Raman imaging of cellular processes
}

Jun Ando ${ }^{1,2}$, Almar F. Palonpon ${ }^{1}$, Mikiko Sodeoka ${ }^{2,3}$ and Katsumasa Fujita ${ }^{1,2}$

${ }^{1}$ Department of Applied Physics, Osaka University, Suita, Osaka, Japan

${ }^{2}$ AMED-CREST, AMED

${ }^{3}$ Synthetic Organic Chemistry Laboratory, RIKEN, Wako, Saitama, Japan

Corresponding author: Fujita, Katsumasa (fujita@ap.eng.osaka-u.ac.jp)

\begin{abstract}
Raman scattering microscopy provides information about the distribution and chemical state of molecules in live cells without any labeling or modification. In recent years, the imaging speed of Raman microscopy has improved greatly owing to the development of instruments that can perform parallel acquisition of Raman spectra from multiple points.
\end{abstract}


In this article, we review recent advances in high-speed hyperspectral Raman imaging and its application to observe various biological processes such as cell mitosis, apoptosis and cell differentiation. Furthermore, we discuss the recent progress in Raman tags for the specific observation of bioactive small molecules in complex biological systems, including the development of organelle-specific probes, imaging of lipid rafts in an artificial monolayer membrane and application as a structure-sensitive tag.

\section{Introduction}

Raman spectroscopy optically addresses each information of molecular vibrations, thereby directly detecting and identifying molecular species in a sample without any labeling or modification. It has been widely used in the measurement of biological samples, especially for identification of molecular species and analysis of molecular conditions such as structures and redox states [1]. At the start of the 1990s, Puppels et al. performed one of the earliest Raman microspectroscopic observation of molecules in living biological cells [2]. Since then, a variety of researches on Raman spectroscopy toward biological and biomedical applications has been conducted, such as analysis of metabolic activity in mitochondria [3], observation of anti-cancer drugs [4] and cancer diagnosis in tissues [5].

Imaging with Raman spectroscopy has become a valuable analytical instrumentation for bioimaging. However, Raman imaging at high temporal resolutions has been technically challenging because Raman spectroscopy requires a long exposure time to detect the weak Raman scattering signals. Conventional laser scanning Raman microscopy usually requires a total imaging time of several hours to one day to get a cell image. High-speed Raman microscopy is therefore necessary to capture the dynamic behavior of specific molecules in a cell. In recent years, the imaging speed of Raman microscopy has improved greatly due to the development of parallel Raman acquisition systems, which can simultaneously acquire Raman spectra from multiple points in a sample [6-8], allowing observation of various biological processes [9-13]. Besides the development of Raman instrumentation, Raman-tag-based detection methods have rapidly grown as a technique for observing specific small molecules in live cells [14-17], and have 
currently been extended to the observation of lipid membrane [18], organelle-specific Raman marker [19] and analysis of molecular structural change [20].

In this article, we review the current status of high-speed hyperspectral Raman imaging systems, its application for the observation of biological processes, and the recent progress in Raman tags for detecting small molecules inside a cell.

\section{Instrumentation for high-speed hyperspectral Raman imaging}

The developments in instrumentation for high-speed Raman microscopy can be classified into four categories: 1) slit-scanning imaging using line-shaped illumination, 2) multi-focus imaging using two-dimensional array illumination, 3) selective illumination for reduced sampling, and 4) global illumination. The details of each system are summarized as follows.

In slit-scanning imaging, the sample is illuminated with a line-shaped laser and Raman spectra from all points in the line are acquired simultaneously. Figure 1a shows a schematic optical configuration of a slit-scanning Raman microscope [6,8]. The laser beam is shaped into a line using a cylindrical lens and focused into the sample by an objective lens. The scattered Raman signals enter the entrance slit of a spectrophotometer, and around 400 Raman spectra along the line are detected in parallel by using a two-dimensional CCD camera. The line-shaped laser is scanned in one dimension using a galvanometer mirror to obtain an image. Figure $1 \mathrm{~b}$ shows a typical Raman image of a living HeLa cell obtained from the slit-scanning Raman microscope, showing the distribution of cytochrome $c\left(\right.$ Cyto-c) at $753 \mathrm{~cm}^{-1}$, proteins at $1686 \mathrm{~cm}^{-1}$ and lipids at $2852 \mathrm{~cm}^{-1}$, with an image size of $208 \times 328$ pixels [8]. The recording time for this image is about 200 times faster than that of a conventional point-scanning Raman microscope, assuming the same imaging parameters are used. In addition to the gain in imaging speed of slit-scanning configuration, recently, the structured illumination method has been utilized to improve its spatial resolution along the direction parallel to the slit of the spectrophotometer [21]. 
Multi-focus Raman imaging uses a micro-lens array [7] or a diffractive optical element [22] to spatially separate the excitation laser beam into a two-dimensional array of foci. The generated Raman signals from each focus are collected with bundled optical fibers, which have been arranged to the same geometry as the illumination array pattern. The other ends of the optical fibers are rearranged into a line along the entrance slit of a spectrophotometer for parallel detection of the Raman spectra. Various versions of this multi-focus scheme have been reported recently. Okuno et al. placed a pinhole array to obtain confocal imaging capability, which suppresses the background signal. They performed Raman imaging of living yeast cells using 48 foci [7]. Kong et al. used rapid beam scanning to form quasi multi-focus excitations while another galvanometer mirror synchronously maps the Raman spectra from each focus point into different channels of a spectrophotometer [13,23]. Schmälzin et al. acquired an entire Raman image in a single exposure using global illumination and an array of bundled optical fibers. The fibers are coupled to an aberration-corrected spectrophotometer equipped with a large CCD detector, allowing parallel Raman detection from 400 points [24].

In selective illumination for reduced sampling, the spatial information and selection of the sampling points are determined by other optical imaging modalities [25-29]. For rapid Raman-based cancer diagnosis, Kong et al. obtained an auto-fluorescence image of a skin tissue sample [25]. The image was then automatically segmented using an unsupervised segmentation algorithm to select the positions for subsequent Raman measurement. Based on this sampling strategy, 70 segments in the $4 \times 4-\mathrm{mm}^{2}$ region of the tissue were retained for Raman identification of basal cell carcinoma. For micron-sized samples such as polymer beads or bacterial spores, the bright field or phase-contrast image was used to reduce the sampling points [27,28]. The center position of each particle was automatically obtained from these images by a centroid-finding algorithm. The excitation laser was then steered to these coordinates to selectively focus on the particles by using a pair of galvanometer mirrors or a spatial light modulator, which allowed the Raman observation of 80 individual particles at random positions simultaneously [29].

In global illumination, the sample is illuminated as a whole by an excitation laser, and the scattered light from the entire observation area is directly imaged on the CCD 
camera [4,30]. A narrow band pass filter is placed before the CCD to observe the scattering intensity at a particular Raman peak of interest. This configuration has advantage to observe large samples. On the other hand, there are some drawbacks such as difficulty of fabricating tunable, high-throughput, narrow bandpass filter, necessity of using high laser powers to illuminate the entire sample, and existence of a huge background signal due to the global illumination. In recent years, another form of global illumination based on light sheet illumination appeared in the literature. A light sheet perpendicular to the detection axis is employed to illuminate a thin slice of the sample, allowing detection of Raman scattering only at the focal plane while removing out-of-focus signals $[31,32]$. Using this technique, Raman images of lipids and proteins in C. elegans worm were obtained [32].

The instruments discussed above use spontaneous Raman scattering for molecular imaging. In recent years, coherent anti-Stokes Raman scattering (CARS) [33] and stimulated Raman scattering (SRS) [34] have also been actively used for hyper-spectral imaging of biological samples. Camp et al. developed a CARS imaging system using a super-continuum laser that covers the spectral range from 500 to $3500 \mathrm{~cm}^{-1}$ with spectral resolution of $10 \mathrm{~cm}^{-1}$ [35], allowing tissue imaging at a frame rate of $\sim 315$ seconds. Ozeki et al. developed a hyper-spectral SRS imaging system with a wavelength-tunable laser, which covers from 2800 to $3100 \mathrm{~cm}^{-1}$ with spectral resolution of $3 \mathrm{~cm}^{-1}$ [36], enabling tissue imaging at a frame rate of $\sim 30$ seconds, and combined it with an on-line analytical system for increased throughput [37]. To further improve the imaging speed, Liao et al. developed a system that modulates the intensity of broadband pump beam with different frequencies for each color. The Raman spectrum, encoded in the Stokes beam through the stimulated Raman gain process, is retrieved by Fourier transformation, allowing a frame rate of 2.4 seconds with wavenumber resolution of 15 $\mathrm{cm}^{-1}$ and spectral range of $2800-3000 \mathrm{~cm}^{-1}$ [38].

\section{Raman imaging of cellular process}

The improvement of imaging speed in Raman microscopy allows us to observe dynamic molecular behavior under various cellular processes. In 2008, we performed time-lapse 
Raman imaging of cellular cytokinesis, where the images were taken every 5 minutes, with a frame rate of 185 seconds [6]. During cytokinesis, the proteins were found to accumulate at the chromosomes whereas Cyto- $c$ seemed to accumulate at the constricted portions of the cell. We also observed cell mitosis and confirmed the movement and split of the chromosomes from the sequence of Raman images of proteins [9].

High-speed Raman microscopy is also advantageous for the observation of intracellular molecules in dynamic cellular events such as apoptosis in its natural environment [10]. We treated living HeLa cells with Actinomycin D, which acts as an apoptosis-inducer, and performed Raman imaging of the cells every 5 minutes with a frame rate of 80 seconds. Around 25 minutes after induction of apoptosis, the time-series of Raman images revealed the release of Cyto- $c$ from mitochondria to cytoplasm while the corresponding Raman spectra showed that the peak intensity of Cyto- $c$ does not significantly change during this period. Since the Raman peak of Cyto- $c$ is drastically decreased when it is oxidized, the above observation showed that the reduced state of Cyto- $c$ is maintained, indicating that Cyto- $c$ does not change its redox state as it is released from mitochondria during apoptosis.

The increased acquisition speed of Raman microscopy is also beneficial to studies on cell differentiation $[11,12,39,40]$. An example is osteoblast differentiation, which occurs during the early stages of bone formation. Osteoblast is known to form hydroxyapatite (HA) during differentiation. Figure 2a shows the time-lapse Raman images of bone marrow stromal stem cells after inducing differentiation into osteoblast [11]. The Raman images were taken every 4 hours for 24 hours, beginning 5 days after stimulation. We confirmed from the time-lapse Raman images of $\mathrm{PO}_{4}{ }^{3-}$ at $956 \mathrm{~cm}^{-1}$ that the HA is gradually formed in osteoblast. The differentiations of embryonic stem (ES) cells and cancer-derived cells have also been investigated using Raman microscopy $[12,39,40]$. The distributions and variations of Raman spectra were found to change depending on the progress of differentiation (Figure $2 b$ ).

An emerging application of fast Raman imaging is in the study of bacterial spore germination dynamics [13]. Kong et al. performed time-lapse Raman observation of 
Bacillus megaterium spores after addition of nutrition to analyze the distribution of various molecules, in particular, $\mathrm{Ca}^{2+}$ dipicolinic acid (CaDPA), protein and nucleic acid, in the spore during germination. The Raman images were taken every 33 seconds with 30 x 30 pixels using a multifocus scan scheme. From the series of Raman images at $1017 \mathrm{~cm}^{-1}$ showing CaDPA and $1665 \mathrm{~cm}^{-1}$ showing protein, they observed the release of CaDPA from the spore while the amount of protein remained the same.

\section{Raman tag for visualizing small molecules in biological systems}

Biological functions of cells are modulated by the effect of bioactive small molecules. The distribution of bioactive small molecules is an important information to elucidate its functional mechanism; however, specific Raman detection of small molecules has been challenging due to the overlap of its Raman signals with background Raman signals from intrinsic biomolecules. In recent years, the method using Raman tags, such as deuterium, nitrile, azide and alkyne, have been developed to specifically detect small molecules in biological samples. These tags possess common features, including 1) a nearly nonexistent presence in cells, 2) a tiny structure that minimizes the alteration of the molecular properties of the tagged molecule, and 3) a signal peak at the Raman-silent region (1800-2600 $\left.\mathrm{cm}^{-1}\right)$ of biomolecules. In particular, alkyne tag exhibits a high scattering intensity, which allows us to detect low-concentration molecules [15]. In 2011, we developed the alkyne-tag Raman imaging methodology and utilized it to visualize the distribution of nucleic acids incorporated in cells [14]. Furthermore, we investigated the relationship between chemical structure and Raman peak intensity/shift [15] of various alkynyl structures, providing us a guideline for designing alkyne-tagged molecules for Raman imaging. According to this guideline, we visualized ubiquinone, a non-covalent-bonding small molecule, using a diyne tag and a thymidine analogue using an alkyne tag in a cell, thereby achieving dual-color alkyne-tag imaging of small molecules [15].

Alkyne-tag Raman imaging has been found useful in the analysis of lipid membranes, in particular lipid rafts [18]. Lipid rafts are micro-domains in a biological membrane, which are thought to have important biological functions in cells. The domain is 
considered to consist of sphingomyelin (SM) and cholesterol (chol), but an analytical method for the observation of such lipid distribution was lacking. To observe the lipid distribution in raft domains, we synthesized diyne-tagged SM (diyne-SM) (Figure 3a) [41] and measured its Raman spectra (Figure 3b). Raman imaging was performed on a ternary monolayer membrane using DOPC and cholesterol as a model of lipid rafts. Figure $3 c$ shows the Raman image of diyne-SM/DOPC/chol ternary monolayer, showing the micro-domain of lipid raft in an artificial monolayer membrane.

Various biomolecules, such as amino acids, fatty acids and choline, were also observed by alkyne-tag Raman imaging with stimulated Raman scattering (SRS) microscopy [16, 42]. This technique was also used to assess the storage and transport of cholesterol in a CHO cell and a C. elegans worm using a phenyl-diyne tag [43]. To expand the use of alkyne tags for multicolor SRS imaging, Chen et al. reported a method using isotope editing to shift the peak position of alkyne [44]. They replaced one of the two carbons or both of them in alkyne with ${ }^{13} \mathrm{C}$ and observed the distribution of three different alkyne-tagged small molecules in a cell.

Although alkyne tags were originally utilized for imaging small bioactive molecules, it could also be used as a sensitive and specific Raman probe for visualizing cellular organelles. Recently, a mitochondria-selective Raman marker based on alkyne was developed [19]. Figure 3d shows the chemical structure of the probe, named MitoBADY. It consists of bisarylbutadiyne as a sensitive Raman tag and triphenylphosphonium as a mitochondria-targeting moiety. This probe with lipophilic cation accumulates in the negatively-charged mitochondria. Raman imaging performed on HeLa cells treated with MitoBADY showed that the contrast of MitoBADY appeared just after 5 minutes of its introduction and reached a plateau in 30 minutes (Figure 3e). The Raman measurement using MitoBADY allowed the observation of both the distribution of mitochondria and that of intrinsic biomolecules such as lipids and proteins (Figure 3f). Other groups have synthesized a Raman marker for mitochondria and membrane based on the resonance Raman scattering effect to enhance the Raman signals [45]. The developments of organelle-specific Raman markers point to an emerging research trend in Raman microscopy. 
Since Raman tags are generally sensitive to its surrounding chemical structure, they can be utilized as a sensor of the structural state of small molecules. Recently, we demonstrated this sensor using nitrile as a structure-sensitive Raman tag to detect two distinct structural forms of the same small molecule [20]. In this study, carbonylcyanide p-trifluoromethoxyphenylhydrazone (FCCP), a small molecule that contains nitriles and that works as an uncoupler of oxidative phosphorylation in mitochondria, was used. Depending on the $\mathrm{pH}$ value of the surrounding environment, FCCP exists as two different structures with each structure showing a distinguishable Raman peak of nitrile. (Figure $4 a$ ). Figure $4 b$ shows Raman images of a HeLa cell treated with FCCP. The Raman images were reconstructed using the peak intensity of deprotonated FCCP at 2176 $\mathrm{cm}^{-1}$, protonated FCCP at $2230 \mathrm{~cm}^{-1}$ and lipids at $2851 \mathrm{~cm}^{-1}$. The results confirmed that protonated FCCP was localized at lipid droplets in the cell, while deprotonated FCCP was uniformly distributed in the cellular cytoplasm, demonstrating the potential of Raman tags for structure-based imaging of bioactive small molecules.

\section{Conclusion}

In this article, we focused our review on recent developments of Raman microscopy for high-speed imaging of biological samples. Modern parallel Raman spectral acquisition systems have improved the imaging speed to around two orders of magnitude faster than conventional Raman microscopy. Hence, relatively slow biological phenomena, such as cytokinesis, mitosis and cell differentiation, can now be investigated by Raman microscopy, which can provide information about the spatial distribution and chemical status of molecules during these processes. In addition, recent advances in Raman-tag detection method, in particular using alkyne tags, have enabled us to observe biomolecules in complex biological systems with high chemical specificity and sensitivity.

The combination of high-speed Raman imaging with molecular-selective Raman tags opens a whole new dimension to the use of Raman microscopy in cellular biology and biomedical science. The results described in this review, such as organelle Raman marker, molecular structural sensor, and lipid monolayer imaging, are just a sample of the new 
applications driven by these recent advances in Raman instrumentation and methodology, and more exciting applications are expected to follow.

\section{Acknowledgement}

This work was partially supported by JST, AMED, RIKEN, JSPS KAKENHI Grant Number 26600117 (J.A.). 


\section{A}
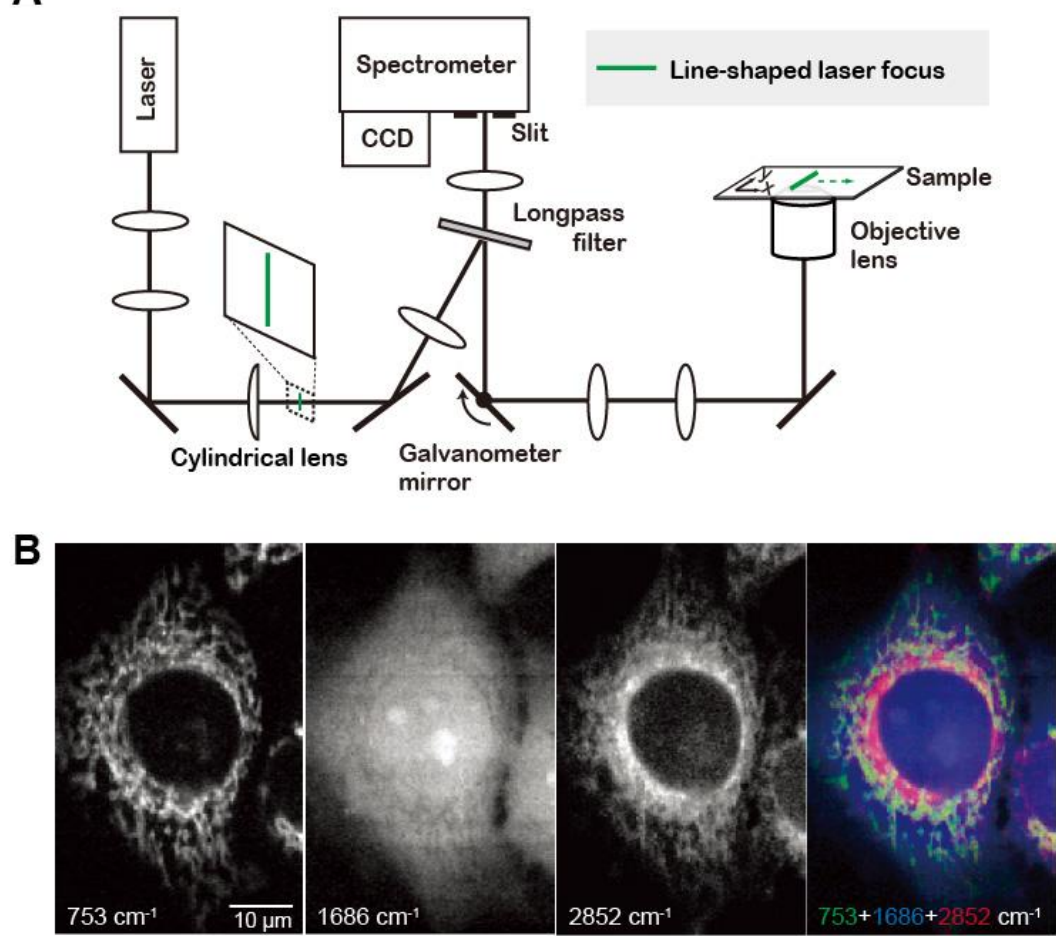

Figure 1: High-resolution molecular imaging of a living HeLa cell using slit-scanning Raman microscopy. (a) Schematic optical configuration of a slit-scanning Raman microscope. (b) Raman images of a living HeLa cell, reconstructed using the peak intensities of cytochrome $\mathrm{c}$ at $753 \mathrm{~cm}^{-1}$, proteins at $1686 \mathrm{~cm}^{-1}$ and lipids at $2852 \mathrm{~cm}^{-1}$. Each image has $208 \times 328$ pixels. Laser intensity was $3 \mathrm{~mW} / \mu \mathrm{m}^{2}$. Exposure time was 5 seconds/line. Source: Adapted from Ref. [8“]. Copyright 2013 Nature Publishing Group. 


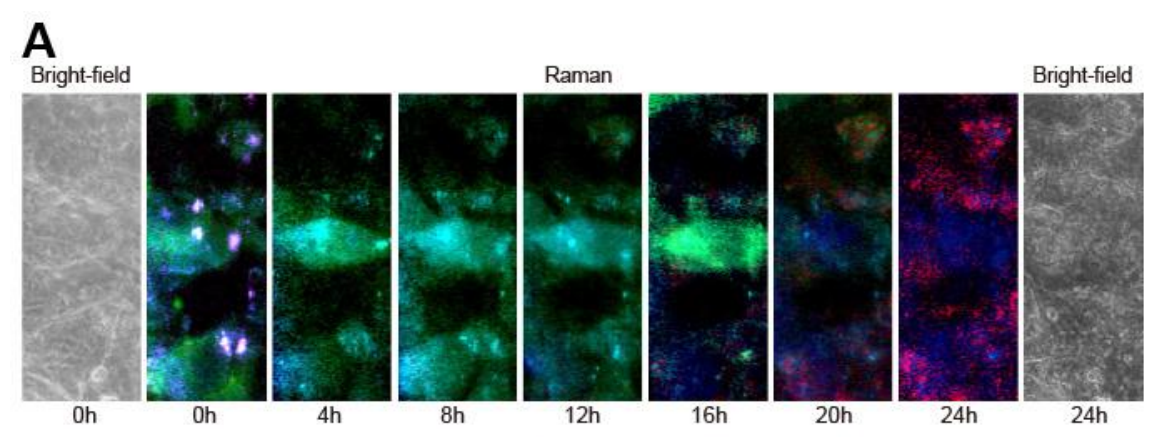

cytochrome $c$ (green, $750 \mathrm{~cm}^{-1}$ ), hydoroxyapatite (red, $956 \mathrm{~cm}^{-1}$ ), $\beta$-carotene (1526 cm-1, magenta), $\mathrm{CH}_{3}$ (blue, $2940 \mathrm{~cm}^{-1}$ )

B
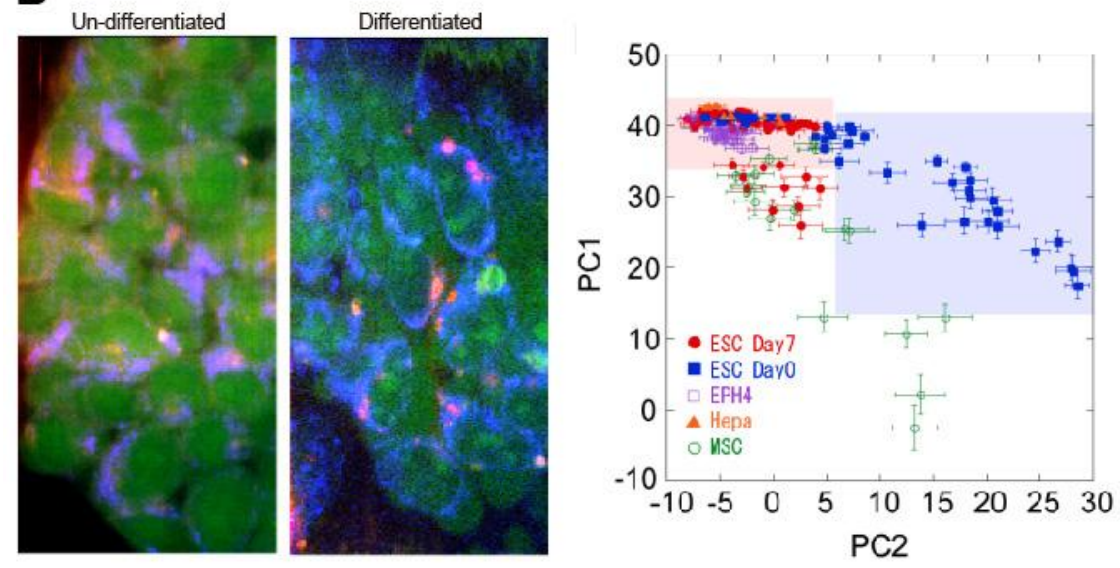

Figure 2: Cell differentiation processes analyzed by slit-scanning Raman microscopy. (a) Time-lapse Raman image of osteoblasts after differentiation. The Raman images were reconstructed using the peak intensities of cyto- $c$ at $750 \mathrm{~cm}^{-1}$ (green), HA at 956 $\mathrm{cm}^{-1}$ (red), $\beta$-carotene at $1526 \mathrm{~cm}^{-1}$ (magenta) and $\mathrm{CH}_{3}$ at $2940 \mathrm{~cm}^{-1}$ (blue). The Raman images were taken every 4 hours for 24 hours, beginning 5 days after inducing differentation [11]. (b) Raman images of undifferentiated and differentiated mouse embryonic stem (ES) cells. The Raman images were reconstructed using the peak intensities of cyto- $c$ at $753 \mathrm{~cm}^{-1}$ (blue), protein at $1660 \mathrm{~cm}^{-1}$ (green) and lipids at 2852 $\mathrm{cm}^{-1}$ (red). The principal component analysis (PCA) of the Raman spectra obtained from the ES cell's nuclei is also displayed. The scores of the Raman spectra in the first two loading vectors, PC1 and PC2, are shown [12]. Source: Adapted from Ref. [11 , 12 ']. Copyright 2015 Nature Publishing Group and 2014 Public Library of Science, respectively. 
A

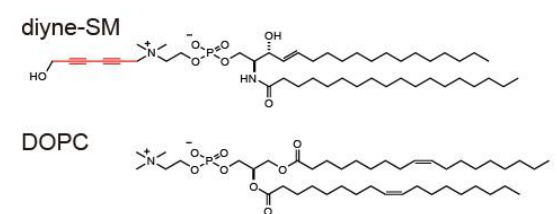

cholesterol

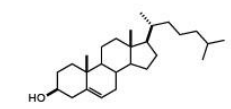

B

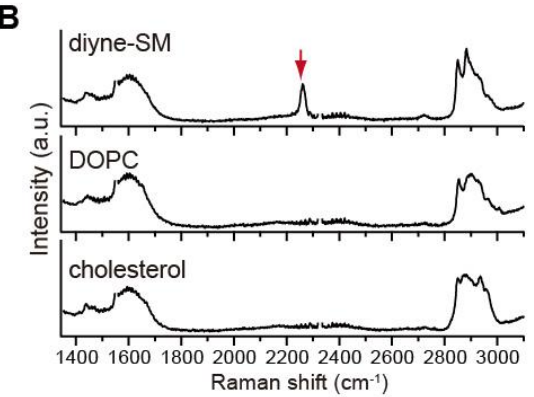

C

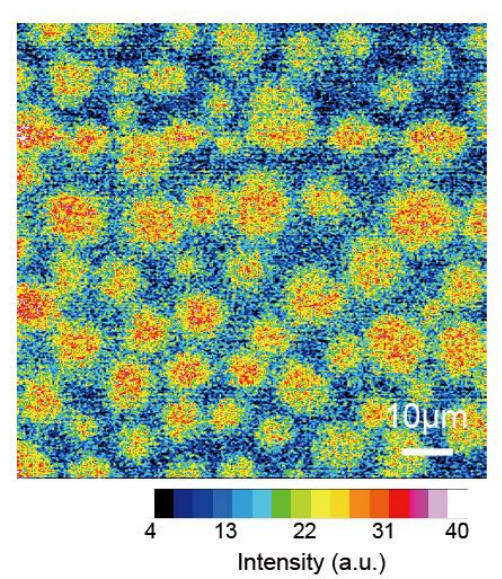

D

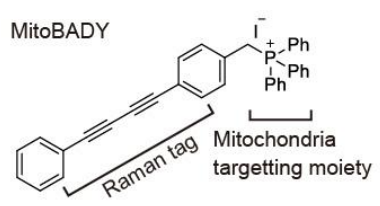

E

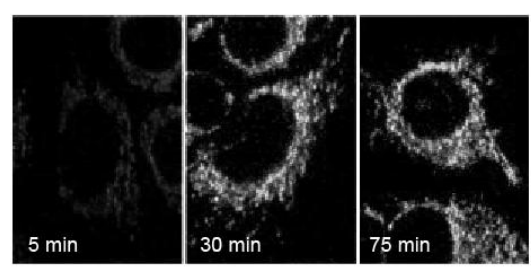

F

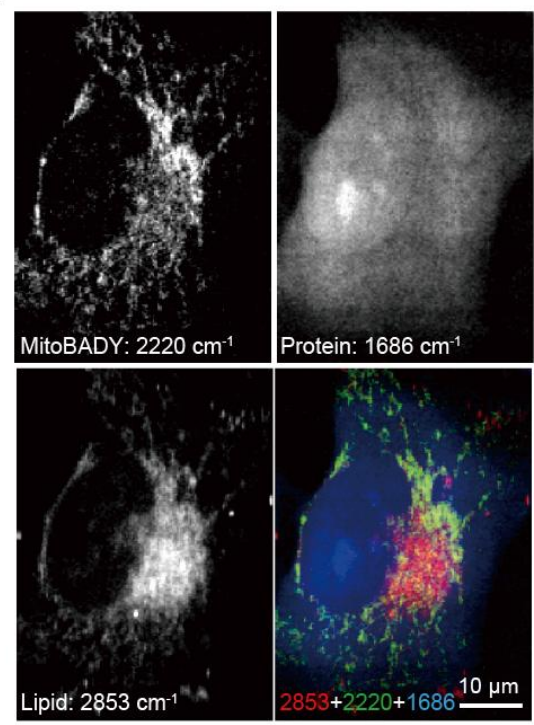

Figure 3: Alkyne-tag Raman imaging for the analysis of lipid rafts in artificial monolayer membrane and development of mitochondria-specific Raman marker. (a) Chemical structures and (b) Raman spectra of diyne-tagged sphigomyelin (diyne-SM), DOPC and cholesterol (chol). (c) Raman image of ternary monolayer membrane of diyne-SM/DOPC/chol. The Raman image was reconstructed using the peak intensity of diyne at $2263 \mathrm{~cm}^{-1}$ [18]. (d) Chemical structure of mitochondria-localized bisarylbutadiyne (MitoBADY). (e) Raman images of living HeLa cells treated with 400 $\mathrm{nm}$ MitoBADY at different incubation times, reconstructed using the peak intensity of bisarylbutadiyne at $2220 \mathrm{~cm}^{-1}$. (e) Raman images of a living HeLa cell with $200 \mathrm{nM}$ MitoBADY, reconstructed using the peak intensities of bisarylbutadiyne at $2220 \mathrm{~cm}^{-1}$, protein at $1686 \mathrm{~cm}^{-1}$ and lipid at $2853 \mathrm{~cm}^{-1}$ [19]. Source: Adapted from Ref. [18 ${ }^{*}, 19^{*}$ ]. Copyright 2015 PNAS and 2015 Elsevier, respectively. 


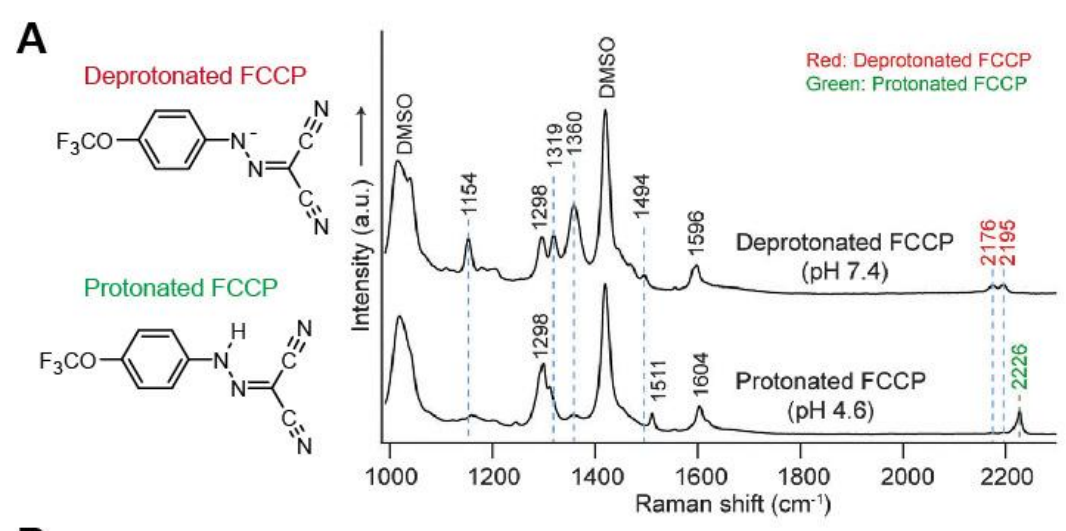

B

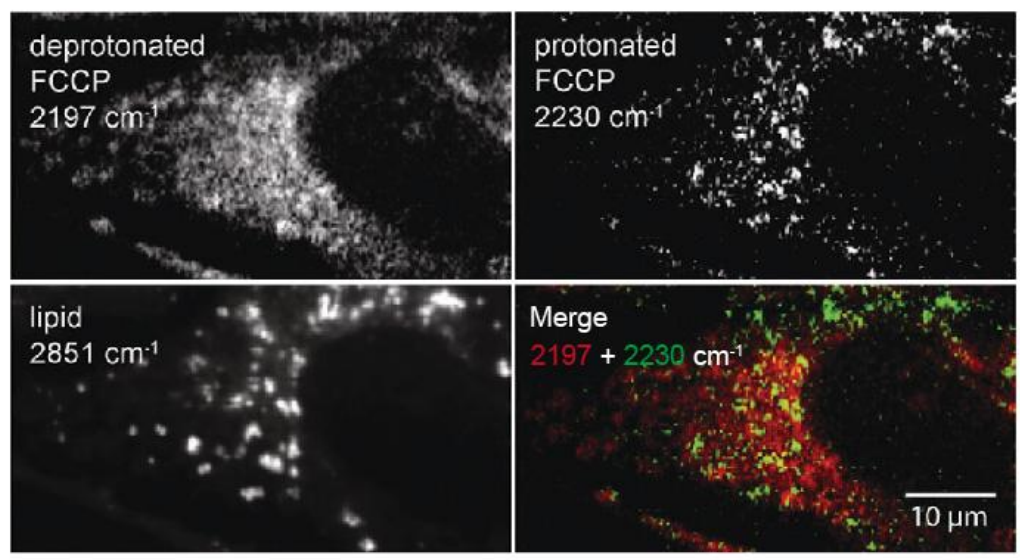

Figure 4: Raman-tag-based intracellular sensor for structural change of small molecule, FCCP (a) Chemical structures of FCCP in its deprotonated and protonated forms, and corresponding Raman spectra obtained at different pH. (b) Raman images of HeLa cell treated with FCCP, reconstructed using the peak intensities of deprotonated FCCP at $2197 \mathrm{~cm}^{-1}$, protonated FCCP at $2230 \mathrm{~cm}^{-1}$ and lipid at $2851 \mathrm{~cm}^{-1}$. Source: Adapted from Ref. [20']. Copyright 2014 the Royal Society of Chemistry. 


\section{References}

- of special interest

- of outstanding interest

1. Lord RC, Yu NT: Laser-excited Raman spectroscopy of bio-molecules I. Native lysozyme and its constituent amino acids. J Mol Biol 1970, 50: 509-524.

2. Puppels GJ, De Mul FFM, Otto C, Greve J, Robert-Nicoud M, Arndt-Jovin DJ, Jovin TM: Studying single living cells and chromosomes by confocal Raman microspectroscopy. Nature 1990, 347:301-303.

3. Huang Y-S, Karashima T, Yamamoto M, Ogura T, Hamaguchi H: Raman spectroscopic signature of life in a living yeast cell. J Raman Spectrosc 2004, 35:525-526.

4. Ling J, Weitman SD, Miller MA, Moore RV, Bovik AC: Direct Raman imaging techniques for study of the subcellular distribution of a drug. Appl Optics 2002, 41: 6006-6017.

5. Stone N, Kendall C, Shepherd N, Crow P, Barr H: Near-infrared Raman spectroscopy for the classification of epithelial pre-cancers and caners. $J$ Raman Spectrosc 2002, 33:564-573.

6. Hamada K, Fujita K, Smith NI, Kobayashi M, Inouye Y, Kawata S: Raman microscopy for dynamic molecular imaging of living cells. J Biomed Opt 2008, 13:044027.

7. Okuno M, Hamaguchi H: Multifocus confocal Raman microspectroscopy for fast multimode vibrational imaging of living cells. Opt Lett 2010, 35:4096-4098.

8. Palonpon AF, Ando J, Yamakoshi H, Dodo K, Sodeoka M, Kawata S, Fujita K: Raman and SERS microscopy for molecular imaging of live cells. Nature protocols 2013, 8:677-692.

$(\cdot)$ This work describes the optical design and protocol to construct a slit-scanning Raman microscope, which allows high-speed hyperspectral Raman imaging of living biological cells.

9. Fujita K, Smith NI, Label-free molecular imaging of living cells. Mol Cells 2008, 26: $530-535$. 
10. Okada M, Smith NI, Palonpon AF, Endo H, Kawata S, Sodeoka M, Fujita K: Label-free Raman observation of cytochrome c dynamics during apoptosis. Proc Natl Acad Sci USA 2012, 109:28-32.

11. Hashimoto A, Yamaguchi Y, Chiu LD, Morimoto C, Fujita K, Takedachi M, Kawata S, Murakami S, Tamiya E: Time-lapse Raman imaging of osteoblast differentiation. Scientific Reports 2015, 5:12529.

(·) This work describes the use of Raman microscopy to observe osteoblast differentiation and revealed the formation of hydroxyapatite in live cells at the early stages of bone formation by time-lapse Raman imaging.

12. Ichimura T, Chiu LD, Fujita K, Kawata S, Watanabe TM, Yanagida T, Fujita H: Visualizing cell state transition using Raman spectroscopy. Plos ONE 2014, 9:e84478.

(·) This work shows the potential of Raman microscopy to visualize the progress of differentiation in embryonic stem cells, revealed by the plot of principal component analysis of the Raman spectra as a change of chemical state in cells.

13. Kong $\mathrm{L}$, Setlow $\mathrm{P}$, Li YQ. Observation of the dynamic germination of single bacterial spores using rapid Raman imaging. J Biomed Opt 2014, 19:011003.

14. Yamakoshi H, Dodo K, Okada M, Ando J, Palonpon A, Fujita K, Kawata S, Sodeoka M: Imaging of EdU, an alkyne-tagged cell proliferation probe, by Raman microscopy. J Am Chem Soc 2011, 133:6102-6105.

15. Yamakoshi H, Dodo K, Palonpon A, Ando J, Fujita K, Kawata S, Sodeoka M: Alkyne-tag Raman imaging for visualization of mobile small molecules in live cells. J Am Chem Soc 2012, 134:20681-20689.

16. Wei L, Hu F, Shen Y, Chen Z, Yu Y, Lin C-C, Wang MC, Min W: Live-cell imaging of alkyne-tagged small biomolecules by stimulated Raman scattering. Nat Methods 2014, 11:410-412.

17. El-Mashtoly SF, Petersen D, Yosef HK, Mosig A, Reinacher-Schick A, Kötting C, Gerwert K. Label-free imaging of drug distribution and metabolism in colon cancer cells by Raman microscopy. Analyst 2014, 139:1155-1161.

18. Ando J, Kinoshita M, Cui J, Yamakoshi H, Dodo K, Fujita K, Murata M, Sodeoka $\mathrm{M}$ : Sphingomyelin distribution in lipid rafts of artificial monolayer 
membranes visualized by Raman microscopy. Proc Natl Acad Sci USA 2015, 112:4558-4563.

$(\cdots)$ This work reports the first imaging of lipid rafts in monolayer membrane using slit-scanning Raman microscopy and alkyne-tag imaging methodology at high spatial resolution and single lipid-layer sensitivity.

19. Yamakoshi H, Palonpona A, Dodo K, Ando J, Kawata S, Fujita K, Sodeoka M: A sensitive and specific Raman probe based on bisarylbutadiyne for live cell imaging of mitochondria. Bioorg Med Chem Lett 2015, 25:664-667.

$(\cdot)$ This work reports the development of mitochondria-selective Raman marker based on alkyne tag and organelle-targeting moiety. Raman microscopy visualized both the distribution of mitochondria and that of intrinsic biomolecules in live cells.

20. Yamakoshi H, Palonpon AF, Dodo K, Ando J, Kawata S, Fujita K, Sodeoka M: Simultaneous imaging of protonated and deprotonated carbonylcyanide p-trifluoromethoxyphenylhydrazone in live cells by Raman microscopy. Chem Commun 2014, 50:1341.

$(\cdot)$ This work shows the potential of Raman tag to analyze the structural state of small molecules in cells. Location-dependent structural change of FCCP, an uncoupler of oxidative phosphorylation in mitochondria that contains nitriles, was observed by Raman microscopy.

21. Watanabe K, Palonpon AF, Smith NI, Chiu LD, Kasai A, Hashimoto H, Kawata S, Fujita K: Structured line illumination Raman microscopy. Nat Commun 2015 6:10095.

22. Samuel AZ, Yabumoto S, Kawamura K, Iwata K: Rapid microstructure characterization of polymer thin films with 2D-array multifocus Raman microspectroscopy. Analyst 2015, 140:1847-1851.

23. Kong L, Zhang P, Yu J, Setlow P, Li Y: Rapid confocal Raman imaging using a synchro multifoci-scan scheme for dynamic monitoring of single living cells. Appl. Phys. Lett. 2011, 98:213703.

24. Schmälzlin E, Moralejo B, Rutowska M, Monreal-Ibero A, Sandin C, Tarcea N, Popp J, Roth MM: Raman Imaging with a Fiber-Coupled Multichannel 
Spectrograph. Sensors 2014, 14:21968-21980.

25. Kong K, Rowlands, CJ, Varma S, Perkins W, Leach IH, Koloydenko AA, Williams HC, Notingher I: Diagnosis of tumors during tissue-conserving surgery with integrated autofluorescence and Raman scattering microscopy. Proc Natl Acad Sci USA 2013, 110:15189-15194.

26. Rowlands, CJ, Varma S, Perkins W, Leach IH, Williams HC, Notingher I: Rapid acquisition of Raman spectral maps through minimal sampling: applications in tissue imaging. J Biophoton 2012, 5:220-229.

27. Qi J, Shih WC: Parallel Raman microspectroscopy using programmable multipoint illumination. Opt Lett 2012, 37:1289-1291.

28. Qi J, Li J, Shih WC: High-speed hyperspectral Raman imaging for label-free compositional microanalysis. Biomed Opt Express 2013 4:2376-2382.

29. Kong L, Zhang P, Setlow P, Li YQ: Multifocus confocal Raman microspectroscopy for rapid single-particle analysis. J Biomed Opt 2011, 16:120503.

30. Papour A, Kwak JH, Taylor Z, Wu B, Stafsudd O, Grundfest W: Wide-field Raman imaging for bone detection in tissue. Biomed Opt Express 2015, 6:3892-3897.

31. Oshima Y, Sato H, Kajiura-Kobayashi H, Kimura T, Naruse K, Nonaka S. Light sheet-excited spontaneous Raman imaging of a living fish by optical sectioning in a wide field Raman microscope. Optics Express 2012, 20:16195-16204.

32. Rocha-Mendoza I, Licea-Rodriguez J, Marro M, Olarte OE, Plata-Sanchez M, Loza-Alvarez P: Rapid spontaneous Raman light sheet microscopy using cw-lasers and tunable filters. Biomed Opt Express 2015, 6:3449-3461.

33. Zumbusch A, Holtom GR, Xie XS: Three-dimensional vibrational imaging by coherent anti-Stokes Raman scattering. Phys Rev Lett 1999, 82:4142-4145.

34. Freudiger CW, Min W, Saar BG, Lu S, Holtom GR, He C, Tsai JC, Kang JX, Xie XS: Label-free biomedical imaging with high sensitivity by stimulated Raman scattering microscopy. Science 2008, 322:1857-1861.

35. Camp CH, Lee YJ, Heddleston JM, Hartshorn CM, Hight Walker AR, Rich JN, Lathia JD, Cicerone MT: High-Speed Coherent Raman Fingerprint Imaging of Biological Tissues. Nat photonics 2014, 8:627-634.

36. Ozeki Y, Umemura W, Otsuka Y, Satoh S, Hashimoto H, Sumimura K, Nishizawa 
N, Fukui K, Itoh K: High-speed molecular spectral imaging of tissue with stimulated Raman scattering. Nat Photonics 2012, 6:845-851.

37. Otsuka Y, Makara K, Satoh S, Hashimoto H, Ozeki Y: On-line visualization of multicolor chemical images with stimulated Raman scattering spectral microscopy. Analyst 2015, 140:2984-2987.

38. Liao C-S, Wang P, Wang P, Li J, Lee HJ, Eakins G, Cheng, J-X: Spectrometer-free vibrational imaging by retrieving stimulated Raman signal from highly scattered photons. Science Advances, 2015, 1:e1500738.

39. Pascut FC, Kalra S, George V, Welch N, Denning C, Notingher I: Non-invasive label-free monitoring the cardiac differentiation of human embryonic stem cells in-vitro by Raman spectroscopy. Biochim et Biophys Acta 2013, 1830:3517-3524.

40. Takanezawa S, Morita S, Ozaki Y, Sako Y: Raman spectral dynamics of single cells in the early stages of growth factor stimulation. Biophys $J 2015$, 108:2148-2157.

41. Cui J, Matsuoka S, Kinoshita M, Matsumori N, Sato F, Murata M, Ando J, Yamakoshi H, Dodo K, Sodeoka M: Novel Raman-tagged sphingomyelin that closely mimics original raft-forming behavior. Bioorg Med Chem 2015, 23:2989-94.

42. Hong S, Chen T, Zhu Y, Li A, Huang Y, Chen X: Live-cell stimulated Raman scattering imaging of alkyne-tagged biomolecules. Angew Chem Int Ed 2014, 53:5827-5831.

43. Lee J, Zhang W, Zhang D, Yang Y, Liu B, Barker EL, Buhman KK, Slipchenko LV, Dai M, Cheng J-X: Assessing Cholesterol Storage in Live Cells and C. elegans by Stimulated Raman Scattering Imaging of Phenyl-Diyne Cholesterol. Scientific Reports 2015, 5:7930.

44. Chen Z, Paley DW, Wei L, Weisman AL, Friesner RA, Nuckolls C, Min W: Multicolor live-cell chemical imaging by isotopically edited alkyne vibrational palette. J Am Chem Soc 2014, 136:8027-8033.

45. Li Y, Heo J, Lim CK, Pliss A, Kachynski AV, Kuzmin AN, Kim S, Prasad PN: Organelle specific imaging in live cells and immuno-labeling using resonance Raman probe. Biomaterials 2015, 53:25-31. 